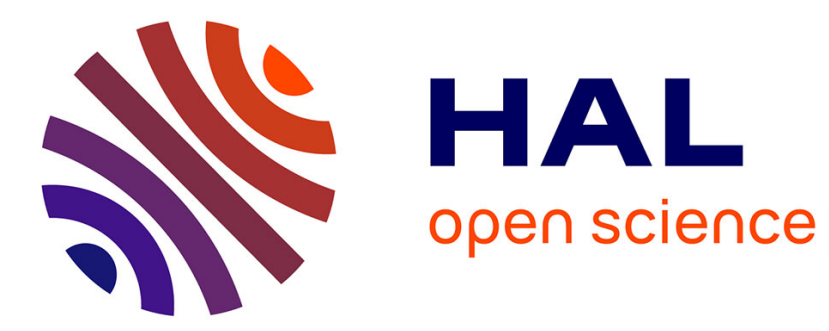

\title{
Note interpretative e testuali alla col. XXII del Papiro di Derveni
}

Walter Lapini

\section{To cite this version:}

Walter Lapini. Note interpretative e testuali alla col. XXII del Papiro di Derveni. KTÈMA Civilisations de l'Orient, de la Grèce et de Rome antiques, 2019, La rhétorique de la diplomatie en Grèce ancienne, 44, pp.171-178. halshs-02444305

\section{HAL Id: halshs-02444305 https://shs.hal.science/halshs-02444305}

Submitted on 17 Jan 2020

HAL is a multi-disciplinary open access archive for the deposit and dissemination of scientific research documents, whether they are published or not. The documents may come from teaching and research institutions in France or abroad, or from public or private research centers.
L'archive ouverte pluridisciplinaire HAL, est destinée au dépôt et à la diffusion de documents scientifiques de niveau recherche, publiés ou non, émanant des établissements d'enseignement et de recherche français ou étrangers, des laboratoires publics ou privés. 

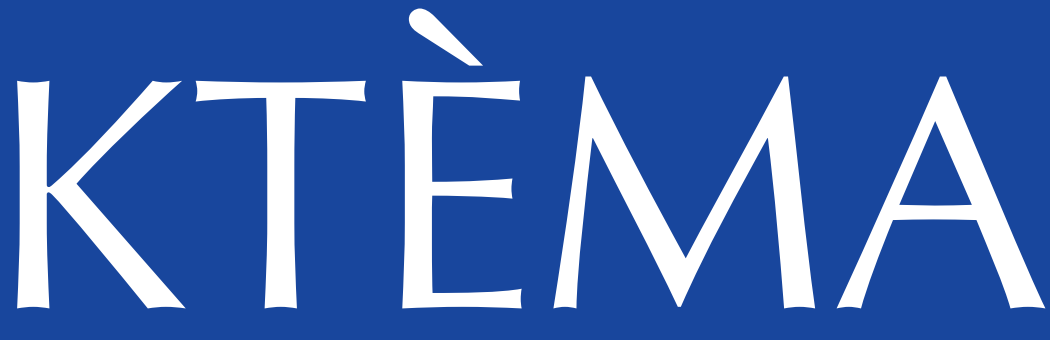

\section{CIVILISATIONS DE L'ORIENT, DE LA GRÈCE ET DE ROME ANTIQUES}

\section{La rhétorique de la diplomatie en Grèce ancienne}

Cinzia BeARzot, Laura Loddo

Laura LoDDo

Cinzia BeArzot

Paolo A. Tuci

Francesca Gazzano

Nicholas D. Cross

Davide Amendola

Jonathan R. W. PraG

Lorenzo CAMPAGNA

Cristina SORACI

Edith Foster

Walter LAPINI

Sylvain PERROT

Gianpaolo Urso

Anne JACQuemin
Political Exiles and Their Use of Diplomacy in Classical Greece

À propos du parallélisme entre deux discours d'ambassade à Sparte (Xén. Hell. V, 2, 12-19 et VI, 1, 4-16). 23

The Speeches of Theban Ambassadors in Greek Literature (404-362 B.C.)................ 33

Greek Ambassadors and the Rhetoric of Supplication. Some Notes ............................ 53

The (Im)balance of Power. Demosthenes' Complex Case

for an Alliance with the Megalopolitans.....

Presbeutikoi and Enteuktikoi Logoi in Hellenistic Interstate Relations.

Some Further Thoughts from an Epigraphical Perspective (c. 306-205 B.C.). 87

\section{Sicile hellénistique et romaine}

I.Sicily, Open Scholarship, and the Epigraphic Landscape of Hellenistic/Roman Sicily.

Trasformazioni urbanistiche in Sicilia alle origini della Provincia.

Riflessioni sul ruolo di Roma.....

Cultes et politique dans la Sicile du i ${ }^{\text {er }}$ siècle av. J.-C.

Le cas de la Vénus Érycine et de la Cérès d'Henna

\section{Varia}

Athens' Political Failures in the Central Chapters of Book 4 of Thucydides 163

Note interpretative e testuali alla col. XXII del Papiro di Derveni

La place de la musique dans la politique culturelle de Téos

dans la première moitié du $\mathrm{II}^{\mathrm{e}}$ siècle avant notre ère

Catilina legatus. Considerazioni su un discusso frammento sallustiano.

La dédicace aux Muses d'Eurydice fille de Sirrhas

(Plutarque, Sur l'éducation des enfants, 20) 


\section{Note interpretative e testuali alla col. XXII del Papiro di Derveni*}

Riassunto-. L'articolo si occupa di alcuni punti difficili della col. XXII del Papiro di Derveni (1) r. 4: il testo

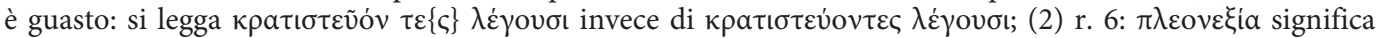

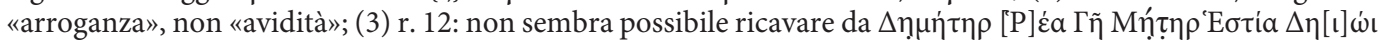
un esametro regolare di tipo letterario: la quantità lunga dell'alpha finale di 'Eotía rende pressoché inutile ogni tentativo in questo senso; (4) rr. 13-14: si intenda «(Orfeo) chiarirà ciò quando sarà arrivato ai versi (dedicati all'argomento in questione)».

Parole-chiave-. Papiro di Derveni, commentari antichi, orfismo, Demetra-De(i)̀̀

Abstract-. This essay deals with some difficult points of Derveni Papyrus Col. XXII: (1) 1. 4: the text

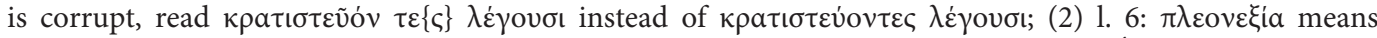

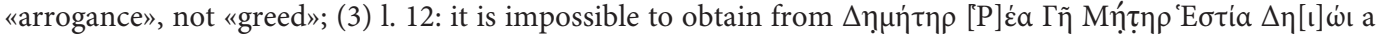
regular hexameter, as the long quantity of the final alpha of 'Eotía nullifies any effort in this direction; (4) ll. 13-14: shall be understood "Orpheus will clarify this when he comes to the verses (devoted to this subject)".

KeYwords-. Derveni papyrus, ancient commentaries, orphism, Demeter-De(i)o

Il Papiro di Derveni, contenente i resti di un commento allegorico a un poema orfico, ha suscitato fin dal suo ritrovamento (1962) un vivo e sempre crescente interesse filosofico, antropologico e linguistico, oltreché, ovviamente, paleografico ${ }^{1}$. Sono stati invece trascurati gli aspetti formali del testo, che pure versa in evidenti condizioni di precarietà. Il fatto che solo poche decine di anni separino l'originale dalla copia ha frenato la mano dei filologi, quasi che esistesse una proporzione fissa tra il passare del tempo e il dosaggio delle corruttele. La realtà è che le corruttele possono pullulare anche in copie vicinissime alla stesura autografa. È ovvio dunque - ma non sarà superfluo ricordarlo - che il critico deve ragionare in base alla ratio e alla res ipsa, e non su

$\left.{ }^{*}\right)$ Devo a Francesca Gazzano suggerimenti importanti, a dimostrazione che l'intelligenza non conosce confini di Fachgebiet.

(1) La prima edizione effettivamente utilizzabile del Papiro di Derveni è quella di R. Merkelbach 1967. L'editio princeps è uscita molto più tardi, nel 2006, per opera di T. Kouremenos, G. M. Parássoglou e K. Tsantsanoglou (KPT 2006). Fra l'una e l'altra si collocano LAKs-Most 1997 (traduzione), JANko 2001 (traduzione), JANko 2002 (testo greco e traduzione), Jourdan 2003 (testo greco basato su JANKo 2002, traduzione, note di commento talvolta anche molto approfondite) e Betegh 2004 (testo greco basato su Janko 2002, traduzione, ampio e pregevole commento). Dopo la princeps si segnalano le edizioni di BERNABÉ 2007 (testo greco con note critiche ricche di bibliografia e loci similes), LAKs, Most 2016 (testo greco e traduzione); Котwick 2017 (testo greco di Janko, diverso in più punti da Janko 2002, traduzione, ampio e intelligente commento). Ancora utilissimo, come studio panoramico sui punti più delicati dell'opera, è il volume del 1997 curato da A. Laks e G. Most (LAKs, Most 1997). 
astratti e non riscontrabili calcoli probabilistici. Nelle righe che seguono esporrò brevemente alcune proposte congetturali ed esegetiche ai primi 14 righi della col. XXII, che riporto qui sotto nel testo e con la traduzione di M.E. Kotwick:

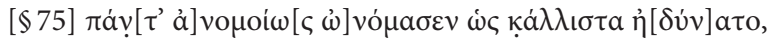

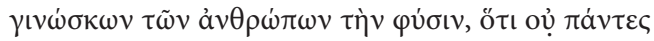

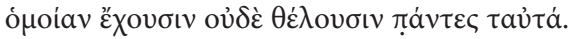

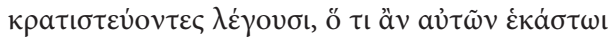

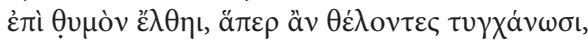

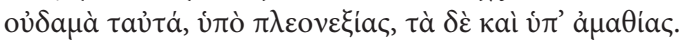

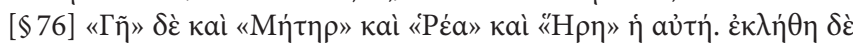

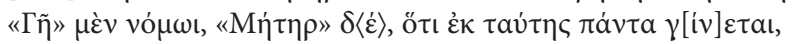

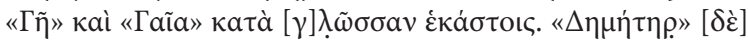

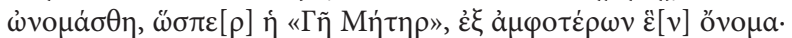

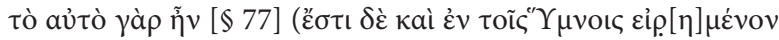

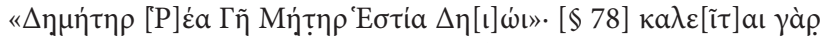

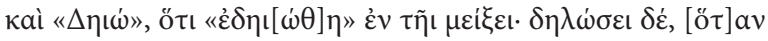

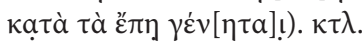

[\$75] (Orpheus) benannte in bestmöglicher Weise alles unterschiedlich, weil er erkannte, dass nicht alle Menschen die gleiche Natur haben und auch nicht alle dasselbe wollen: Wenn sie große Macht haben, sagen sie, was einem jedem von ihnen in den Sinn kommt, was immer sie gerade wollen, niemals dasselbe, aus Gewinnsucht, aber auch aufgrund von Unkenntnis. [\$76] «Ge» («Erde»), «Meter» («Mutter»), «Rhea» und «Hera» ist dieselbe. «Ge» («Erde») wurde sie aus Konvention genannt, «Meter» («Mutter») aber, weil aus ihr alles entsteht, «Ge» und «Gaia» nach der jeweiligen Mundart. Und sie heißt «Demeter» wie «Ge-Meter» («Erd-Mutter»), aus beiden (ensteht) ein Name - denn er (der Name) war derselbe. [\$77] (Und auch in den Hymnen heißt es: «Demeter Rhea Ge Meter Hestia Deio». [\$78] Denn sie wird auch «Deio» genannt, weil sie bei der Vereinigung vernichtet (edeiothe) wurde. Es wird klar werden, wenn sie (sc. die Vereinigung) im Gedicht vorkommt $)^{2}$.

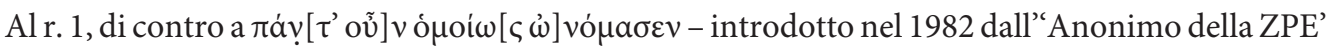

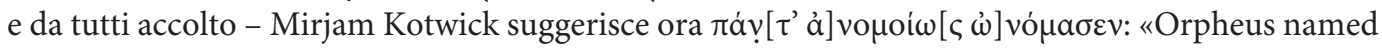
everything differently» ${ }^{3}$. Nel primo caso si verrebbe a dire che Orfeo si oppone al particolarismo degli óvó $\mu \alpha \tau \alpha$; nel secondo, che lo accetta e lo promuove. Ora, se la difference fosse espressa con

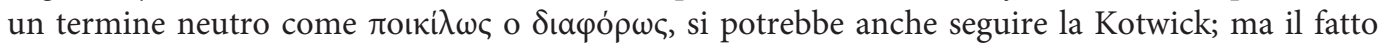
che ai rr. 2-3 la non-ópotótᄁৎ delle nature umane venga presentata come un handicap e non come un vantaggio conferisce ad àvouoi $\omega \varsigma$ una nuance peggiorativa che lo rende poco compatibile con

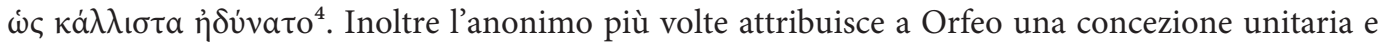
sintetica del linguaggio: si veda la contrapposizione fra la varietà e il termine singolo in XVIII 7-9

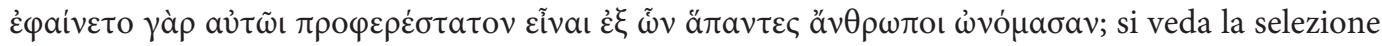

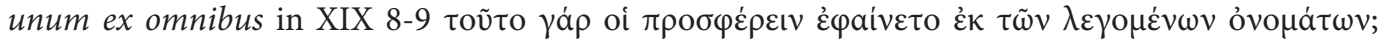

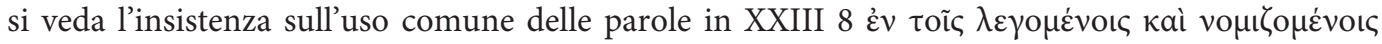

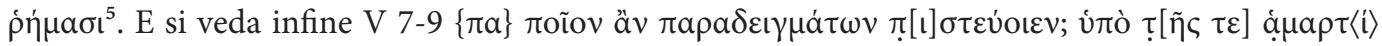

(2) Cf. Kotwick 2017, p. 94-97 = Bernabé 2007, p. 244-247.

(3) Kотwick 2016, p. 2; per la traduzione tedesca (p. 95, ma anche p. 302) si veda sopra nel testo. La Kotwick è ora seguita da SALAmone 2016, p. 98 e 231.

(4) Se si adotta ả] vouoí $\omega$ [ $\varsigma$ bisogna rinunciare a oũv. Ma non è un gran danno: come la Kotwick stessa osserva (2016,

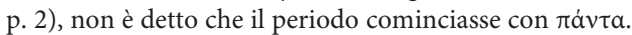

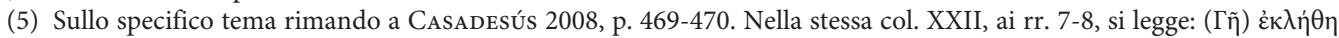

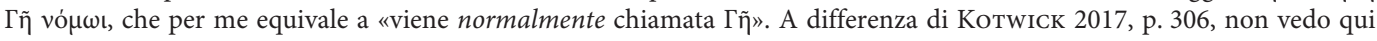
una contrapposizione vó $\mu \omega t-\varphi v ́ \sigma \varepsilon$. 


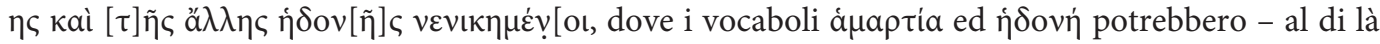
della ben evidente facies etico-religiosa - inquadrarsi anch'essi in una problematica linguistica ${ }^{6}$ :

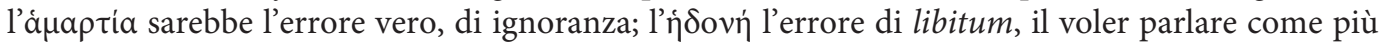
piace, a capriccio ${ }^{7}$.

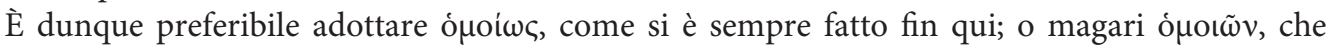

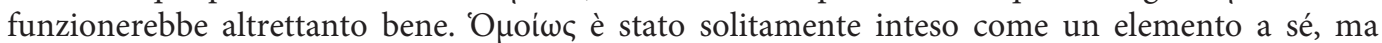
potrebbe anche riferirsi a un dativo espresso o sottinteso, a indicare la conformità di Orfeo a scelte altrui o sue proprie: «conformemente (a e.g. Omero) Orfeo chiamò...», oppure "conformemente

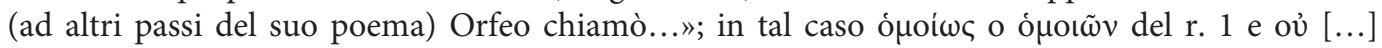
ónoíav dei rr. 2-3 non costituirebbero un nesso analettico, ma solo una falsa anafora. Anche il Casadesús, che parafrasa «adoptó una actitud activa que lo llevó a denominar a todos los seres de la manera más bella y adecuada a su naturaleza particular", sembra presupporre un uso non

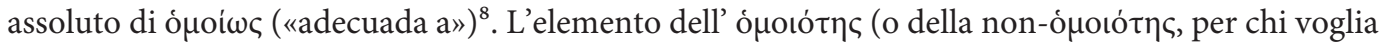
seguire la Kotwick) risulta comunque importantissimo, ed è singolare che V. Piano lo abbia escluso dalla traduzione che fornisce del passo nel suo sorprendente libro sul Papiro di Derveni del 2016: «dunque denominò ogni cosa come meglio poteva, conoscendo la natura degli uomini, poiché non l'hanno tutti uguale»" ${ }^{9}$ traduzione in cui si rileva anche una poco corretta interpretazione di ö $\tau$ เ (evidentemente dichiarativo, non causale) ${ }^{10}$.

$\Pi \lambda \varepsilon \varepsilon_{0} \varepsilon \xi i a$ del r. 7 vuol dire prevaricazione, arroganza, ma poiché il testo così come è trasmesso non giustifica tale valore del termine, la traduzione che in genere si adotta è «avidità». Che però

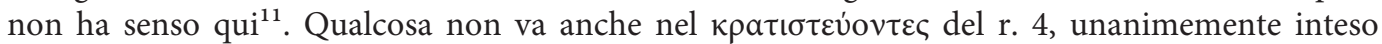
«(gli uomini) quando hanno il potere» ${ }^{12}$. Il nesso parola-potere è suggestivo e suona bene, ma la prerogativa del dire ciò che viene in mente (che è altra cosa dalla parrhesia) temo non dipenda

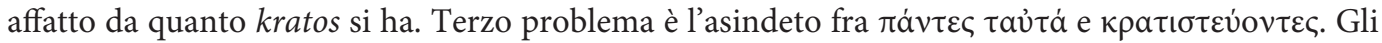

(6) Già Frede 2007, p. 15 e Rangos 2007, p. 39 avevano individuato elementi di teoria del linguaggio in questo passo.

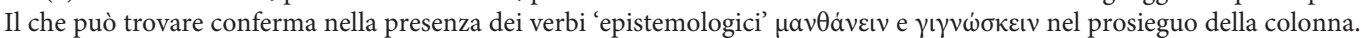

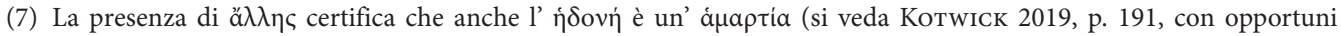
confronti fra la col. V e il Gorgia di Platone). Alcuni danno ad ä $\lambda \lambda \eta \varsigma$ un valore autonomo: cf. Jourdan 2003, p. 5 «la faute, et le plaisir sous toutes ses formes»; LAKs-Most 2016, p. 385 «overcome both by error and by something else, pleasure (or: by the other pleasure)»; Котшіск 2017, p. 137; altri trasformano ä $\lambda \lambda \eta \varsigma$, di fatto, in un kai rafforzato: cf. JANKo 2001, p. $20=2002$, p. $11=$ TsANTSANOGLOU-PARÁSSOGLOU 2006, p. 130 «overcome by both error and pleasure as well»; LAKS 2007, p. 161 «by error and also by pleasure»; altri traducono come se ä $\lambda \lambda \eta \varsigma$ neppure ci fosse: cf. BETEGH 2004(a), p. 361;

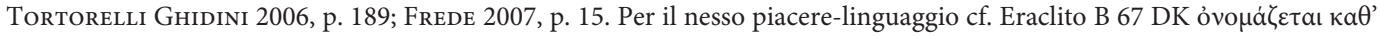

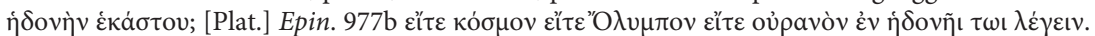

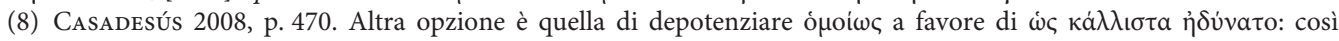
Оввікк 1994, p. $122=1997$, p. $48=2003$, p. $185=2010$, p. 20: «likewise in the noblest possible way».

(9) Piano 2016(b), p. 118 n. 120.

(10) E così infatti i traduttori intendono; tutti tranne TorTorelli GHidini 2006, p. 223: «dunque denominò allo stesso modo tutte le cose come meglio poteva conoscendo la natura umana, poiché non tutti l'hanno uguale» (scil. la natura), da cui la Piano sembra generosamente attingere.

(11) «Greed» (Laks-Most 1997, p. 20 = 2016, p. 423; Obbink 1994, p. $122=1997$, p. 48; Janko 2001, p. $29=2002$, p. 45; Betegh 2004(a), p. 47; McKirahan 2010, p. 466; Tsantsanoglou, Parássoglou 2006, p. 137; Rangos 2007, p. 39), «convoitise» (Jourdan 2003, p. 22); «avidità» (Tortorelli Ghidini 2006, p. 223). La Piano passa dall'«avidità» della traduzione (2016(b), p. 118 n. 120) alla «cieca avidità» della parafrasi (2016(b), p. 121), con un'inutile enfasi che radicalizza il concetto e quindi aggrava il fraintendimento.

(12) «When they have power» (LAKs-Most 1997, p. 20 = 2016, p. 423 = McKirahan 2010, p. 466; similmente Betegh 2004(a), p. 47, e Tsantsanoglou, Parássoglou 2006, p. 137; Ramelli 2007, p. 919); "when they are in a position of роwеr» (Оввілк 1994, p. $122=1997$, p. 48); «when they are very powerful» (Janko 2002, p. 45); «while they are strong and healthy» (RANGOS 2007, p. 39); "quand ils sont les plus puissants» (Jourdan 2003, p. 22); «lorsqu'ils sont puissants» (SAlamone 2016, p. 99); «wenn sie große Macht haben», «die Mächtigsten», «in Machtpositionen» (Kotwick 2017, p. 97 e 303); «se sono potenti» (Tortorelli GHIdini 2006, p. 223). 
asindeti sono frequenti nel Papiro di Derveni (cf. VIII 6, XII 3, XIV 5, XIV 7, XV 7, XV 8, XVI 7, XIX 3, XIX 11, XXI 5-8, XXIII 1, XXIII 12, XXIV 4, XXV 9-10), ma presentano quasi sempre una funzione esplicativa, ora perché si attaccano direttamente alle citazioni da 'Orfeo' ora perché ne riprendono e commentano a breve distanza gli ipsa verba ${ }^{13}$. Tsantsanoglou indica VI 3, 4, 7, 8 e XXII 4 come i soli casi in cui l'asindeto «does not seem to serve any rhetorical purpose and strengthens the impression of carelessness» ${ }^{14}$; pertanto se mettiamo da parte le prime sei colonne, troppo incerte, e se prescindiamo dai due casi problematici di XIX 3 e XXI 5-8, su cui intendo

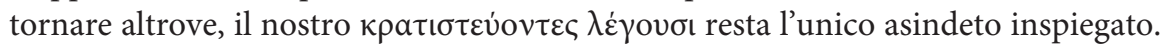

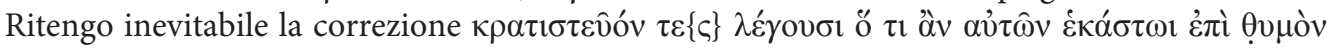
है $\lambda \theta \eta$ : «e dicono che il meglio è ciò che (di volta in volta) viene loro in mente» ${ }^{15}$. Il lieve ritocco risolve tutti e tre i problemi in un colpo solo: (1) elimina l'asindeto; (2) elimina la bizzarra idea che chiamare le cose ad libitum dipenda dal trovarsi in una posizione di potere; (3) dà al termine $\pi \lambda \varepsilon$ ov $\varepsilon \xi i ́ \alpha$ il più plausibile senso di «arroganza» ${ }^{16}$. E infatti arroganza il credere migliori i nostri

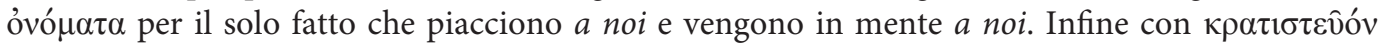

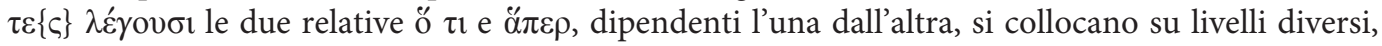
perdendo il loro aspetto di mera ripetizion ${ }^{17}$; e anche il passaggio dal singolare ó $\tau \imath$ al plurale ö $\pi \varepsilon \rho$

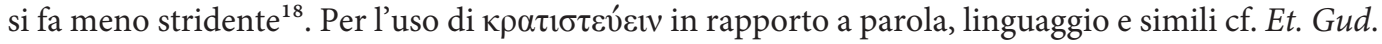

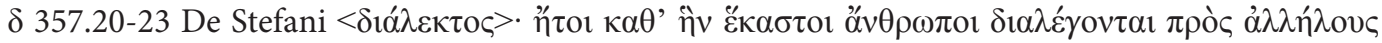

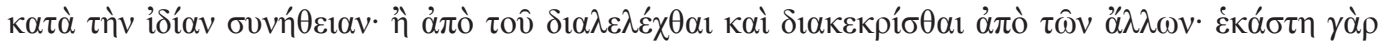

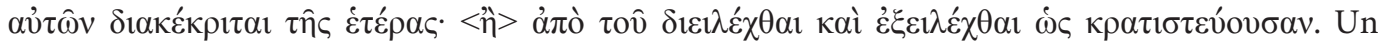

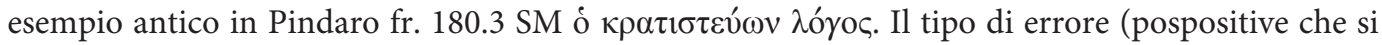

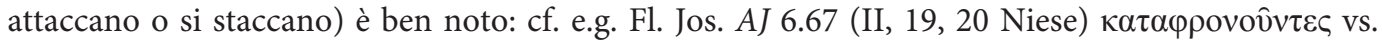

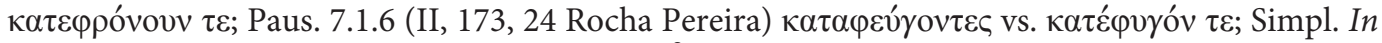
Aristot. Cat. VIII, 5, 28 Kalbfleisch ióv $\tau \varepsilon \varsigma$ vs. oî́v $\tau \varepsilon$. Anche l'Echoschreibung dei due precedenti $\pi \alpha ́ v \tau \varepsilon \varsigma$ (rr. 2 e 3 ) può aver avuto la sua parte di responsabilità.

Al r. 10 accetterei il ritocco $\dot{\omega} \sigma \pi \varepsilon[\rho] \varepsilon i ́$ per $\dddot{\sigma} \sigma \varepsilon \varepsilon[\rho] \dot{\eta}$, proposto con forse eccessiva circospezione

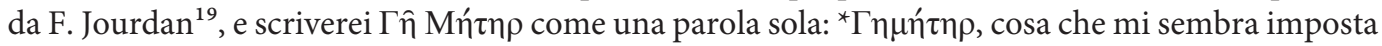

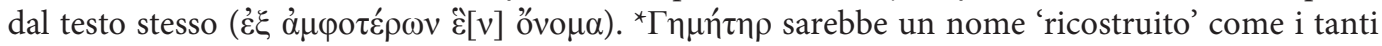

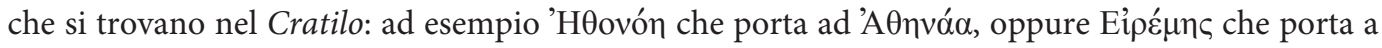

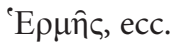

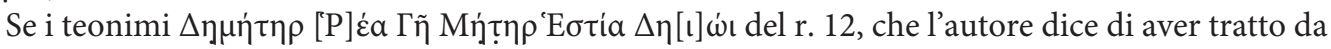

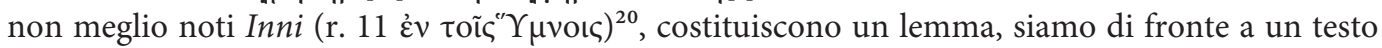

(13) Котшіск 2017, p. 121, 144, 169, 298, 303. L'uso si diffonderà largamente nella tarda tradizione ipomnematica: cf. e.g. Sedley, Bastianini 1995, p. 257; Wittwer 1999, p. 67; Tsantsanoglou 2006, p. 14.

(14) Tsantsanoglou 2006, p. 14.

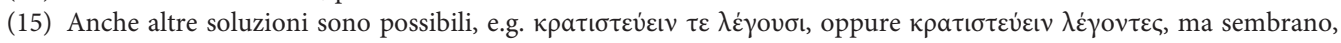
almeno in astratto, più costose. La cosa che veramente importa è che il verbo sia riferito all'oggetto del «dire» e non, assurdamente, a coloro che dicono.

(16) «Arrogance» già in FREDE 2007, p. 15; RAMELLi 2007, p. 919 («spirito di sopraffazione»), e SALAMONE 2016, p. 231 e 258; e ancora prima in BetEGH 2004(a), p. 47, anche se solo come soluzione di riserva: «greed (or: arrogance)».

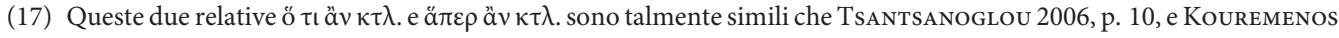
2006 , p. 241, le mettono in alternativa come possibili varianti d'autore. Due relative dipendenti l'una dall'altra si ritrovano

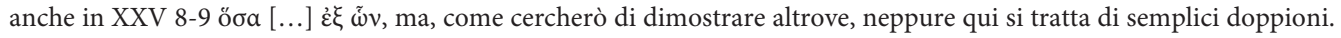

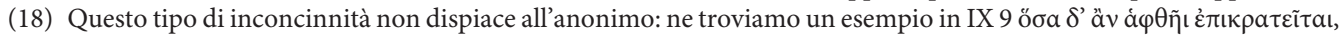

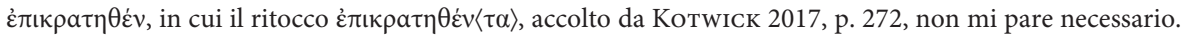

(19) Jourdan 2003, p. 92 n. 2.

(20) Questo è uno dei rari casi in cui l'anonimo chiama in causa un testo diverso dal poema orfico di cui viene svolgendo il commento; e anche la formula introduttiva è atipica: cf. BETEGH 2004(b), p. 40. 
ametrico. D. Obbink deduce dal secondo iota (abusivo) di $\Delta \eta \iota^{\prime} \iota^{21}{ }^{21}$ un'origine vocativale di tutto il

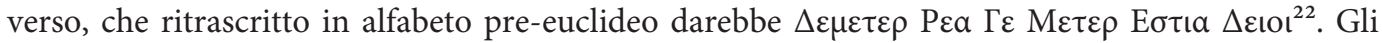
studiosi hanno fatto vari tentativi per ripristinare un esametro corretto: J. S. Rusten misura il nome 'Péa spondaicamente $\left(\mathrm{P} \varepsilon \dot{\alpha} \alpha={ }^{2} \mathrm{P} \varepsilon \dot{i} \alpha\right)^{23}$, mentre D. Obbink e N. Kazansky lo comprimono in un unico longum; il che rende necessario integrare due sillabe brevi o una sillaba lunga in qualche punto del

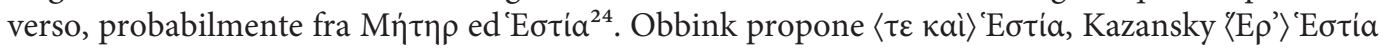
$={ }^{\prime \prime} \mathrm{H} \rho(\eta)^{\circ} \mathrm{E} \sigma \tau i \alpha^{25}$.

Sulla questione nominativo-vocativo va ricordato che lo iota 'analogico' dopo omega è diffusissimo in ogni epoca e in ogni contesto: cf. POxy. 3372 col. II, rr. 32-33 $\lambda \dot{\varepsilon} \gamma \omega$ i; POxy. 3376

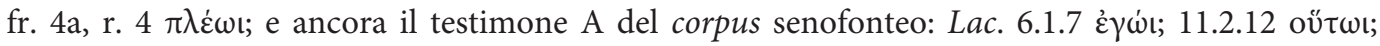

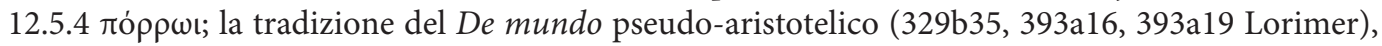
eccetera. Perciò il minimo che si può dire della lettura vocativale di Obbink è che si basa su un

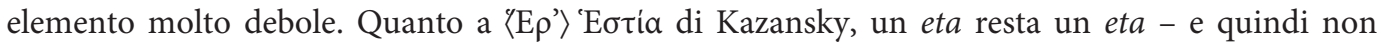
soggetto a elisione - anche se è scritto epsilon. Il Kouremenos afferma che Eơtía ha lo iota lungo, ma non è vero: di norma è breve ${ }^{26}$. Il $\tau \varepsilon$ kai di Obbink è accolto da Janko nell'apparato, da Bernabé nel testo ${ }^{27}$. Bene Janko nel 2002 e bene Bernabé nel 2004 (PEG II, 1: edizione del frammento), male Bernabé nel 2007 (PEG II, 3: edizione del P. Derveni) ${ }^{28}$ : una cosa infatti è il quoter, un'altra il quotee $e^{29}$. Ammesso, e per nulla concesso, che $\tau \varepsilon$ kai sia lezione originale degli Inni, non è dimostrabile che la sua scomparsa dal papiro sia da imputare allo scriba piuttosto che all'autore, il quale peraltro in questo caso non dispone il lemma su un intero rigo di scrittura come usa fare con i full-verse lemmata. Il Bernabé infine non si accorge che nonostante l'aggiunta di $\tau \varepsilon$ kaí il suo esametro continua a non tornare: l'alpha di'Eotía dovrebbe essere o breve o in iato, e invece non è né breve né in iato ${ }^{30}$. Anche l'ottima Mirjam Kotwick fa confusione su questo punto: «Kouremenos

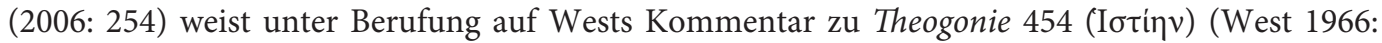
293-294) darauf hin, dass das । in 'Eotia lang ist und somit auch die Einfügung des $\tau \varepsilon$ kai den Vers nicht heilt. Diesem Vorbehalt ist zu entgegnen, dass die Langmessung des I zwar neben der Hesiod-Stelle auch für die Odyssee gilt (14.159'Ioтin [...]), nicht aber für die Homerischen Hymnen

(21) Sulla legittimità del primo dei due iota si può dubitare. La grafia $\Delta \eta \iota \dot{\omega}$ si incontra spesso nelle tradizioni manoscritte, e viene regolarmente corretta in $\Delta \eta \dot{\omega}$. BREMMER 2006, p. 27, propone di recuperarla, in base all' «absolutely trustworthy witness» costituito appunto dal Papiro di Derveni, che presenta $\Delta \eta \iota \dot{~ c o n ~ i o t a . ~ L ' i s t a n z a ~ d i ~ B r e m m e r ~ e ̀ ~ t a n t o ~}$ giusta quanto è sbagliata la motivazione. I papiri letterari, di Derveni o non di Derveni, non sono mai assolutamente affidabili. Solo gli originali lo sono - e forse neppure loro. Comunque sia, l'autore si serve della grafia $\Delta \eta \iota \dot{\omega}$ per facilitare

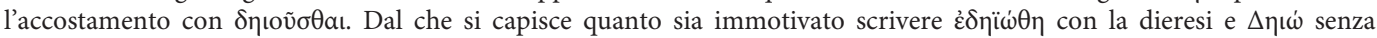
dieresi, come fanno Tsantsanoglou, Parássoglou 2006, p. 105 e 137; Brisson 2009, p. 30 n. 26; Piano 2016(a), p. 12; LaKs, Most 2016, p. 424.

(22) Оввімк 1994, p. 123 n. 43.

(23) Rusten 1985, p. 137 n. 34.

(24) Оввіnк 1994, p. 123 n. 43.

(25) La notizia è in Оввіnк 1994, p. 123 n. 43

(26) Kouremenos 2006, p. 254. È lungo in Hes. Th. 454; breve in Hymn. Hom. in Vest. 1, 6; Ap. Rh. 4.693; Theocr. 22.205, ecc.; cf. anche Котwick 2017, p. 309.

(27) Come Janko 2002, p. 44 (in apparato), così anche Bernabé 2004, p. 330, e 2007, p. 245 sceglie il vocativo. Si legga

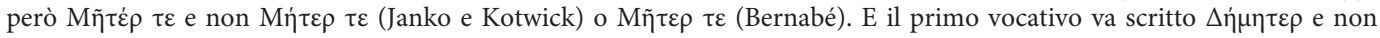
$\Delta \eta \mu \eta \dot{\tau \varepsilon \rho ~(К о т ш і с к ~ 2017, ~ p . ~ 309) . ~}$

(28) Rispettivamente BernabÉ 2004, p. 330, e 2007, p. 245.

(29) Cf. Lapini 2015, p. XVII n. 1, nonché Tosi 1988, p. 52; Dorandi 2010, p. 273ss.; Dorandi 2013, p. 194 e 199ss. Lo stesso Bernabé è consapevole (né potrebbe non esserlo) di questa differenza: cf. 2010, p. 77.

(30) Una controllata alla metrica non guasterebbe anche in altri punti dei PEG, e.g. II.1, Carmina theogonica $141.2 \mathrm{~F}$

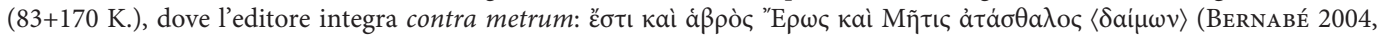

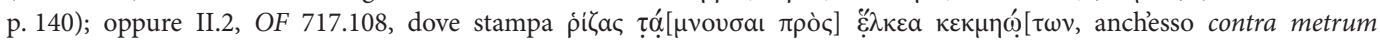
(Bernabé 2005, p. 282). 
(s. 24.1, 6, 10 und 29.1) oder auch das Drama, wo das । kurz gemessen wird ${ }^{31}$. Giusta e doverosa la precisazione sulla brevitas di iota ${ }^{32}$; ma questa brevitas non risolve comunque nulla, finché resta il problema della longitudo di alpha.

Nei suoi davvero unici lavori sul Papiro di Derveni, V. Piano presenta in questo modo i rr. 9-10

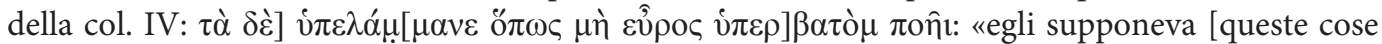
perché (scil. il sole) non] rendesse superabile [la sua ampiezza]» ${ }^{33}$. La filologa molisana ritiene che

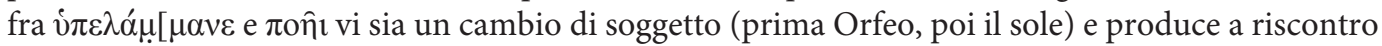
l'analoga metabasis di XXII 13-14, dove, secondo i più, $\delta \eta \lambda \omega ́ \sigma \varepsilon ı$ avrebbe come soggetto Orfeo ${ }^{34}$, e $\gamma \varepsilon \dot{\varepsilon} v \eta \alpha \iota$ Deò ${ }^{35}$ oppure la $\mu \varepsilon \hat{\imath} \xi 1 c^{36}:$ «(Orpheus) will reveal this when, according to his verses, she comes to be»; «il voudra <le> montrer d'après <ces> vers du mois, <lorsqu'elle vient à l'être >»; "chiarirà quando, secondo i versi, sia nata»; «he will show this when in his verses she is born», $\mathrm{e}$

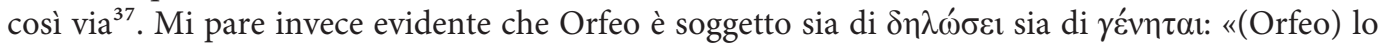
spiegherà quando sarà arrivato ai versi (dedicati a questo argomento)», secondo un ben noto uso di

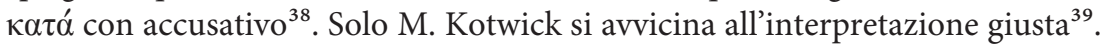

Walter LAPINI Università di Genova

\section{Bibliografia}

Anonimo, 1982, «Der orphische Papyrus von Derveni», ZPE 47, p. 301-312.

Bernabé, A., 2004, Poetae epici Graeci. Testimonia et fragmenta, vol. II, 1: Orphicorum et Orphicis similium testimonia et fragmenta, Monachii et Lipsiae (= PEG II, 1).

Bernabé, A., 2005, Poetae epici Graeci. Testimonia et fragmenta, vol. II, 2: Orphicorum et Orphicis similium testimonia et fragmenta, Monachii et Lipsiae (= PEG II, 2).

Bernabé, A., 2007, Poetae epici Graeci. Testimonia et fragmenta, vol. II, 3: Musaeus, Linus, Epimenides, Papyrus Derveni, indices, Berolini et Novi Eboraci (= PEG II, 3).

Bernabé, A., 2010, “The Derveni Papyrus: Problems of Edition, Problems of Interpretation", in T. Gagos (ed.), Proceedings of the Twenty-Fifth International Congress of Papyrology, Ann Arbor, p. 77-84.

Betegh, G., 2004(a), The Derveni Papyrus. Cosmology, Theology and Interpretation, Cambridge.

(31) Kотwicк 2017, p. 309.

(32) Si veda sopra, n. 25.

(33) Piano 2016(b), p. 78 e 80; cf. anche Lapini 2018(b), p. 54-55.

(34) Così Laks, Most 1997, p. 20 = 2016, p. 425; Janko 2001, p. 29 = 2002; Jourdan 2003, p. 22; Betegh 2004(a), p. 47; Tortorelli Ghidini 2006, p. 223; McKirahan 2010, p. 467. Poco cambia se $\delta \eta \lambda \omega ́ \varepsilon \varepsilon$ viene inteso come equivalente

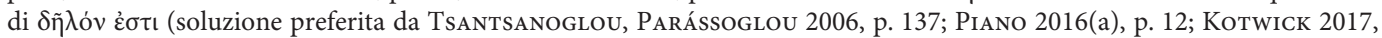
p. 96 e 346$)$.

(35) Cf. Janko 2001, p. 29; 2002, p. 45; Jourdan 2003, p. 22; Betegh 2004(a), p. 47 e 263; Tortorelli Ghidini 2006, p. 223; McKirahan 2010, p. 467; LaKs, Most 2016, p. 425; Piano 2016(a), p. 12.

(36) Cf. Ramelli 2007, p. 919; Котwick 2017, p. 96 e 311. La possibilità è considerata anche da Janko 2002, p. 29 n. 168.

(37) Rispettivamente Janko 2002, p. 45; Jourdan 2003, p. 22; Tortorelli Ghidini 2006, p. 223; Laks, Most 2016,

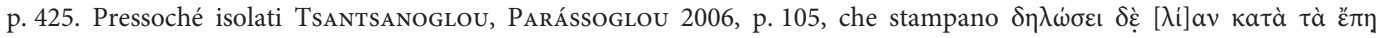
$\gamma \varepsilon v \underline{[}[\tilde{a} v]:$ «it will be made clear that, according to the poems, she had profused parturitions».

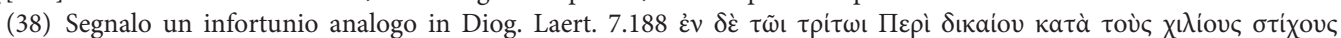

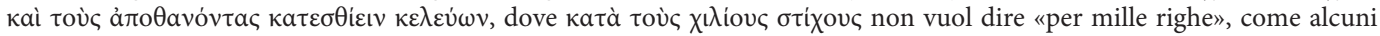
traduttori pensano, bensì «all'altezza del millesimo rigo» (cf. LAPINi 2018(a), p. 243-244 e n. 13-14).

(39) Kотwick 2017, p. 311. 
Betegh, G., 2004(b), "Exegesis in the Derveni Papyrus", in P. Adamson, H. Baltussen, M. W. F. Stone (eds.), Philosophy, Science and Exegesis in Greek, Arabic and Latin Commentaries, London, p. 37-50.

Bremmer, J. N., 2006, «Rescuing Deio in Sophocles and Euripides», ZPE 158, p. 27.

Brisson, L., 2009, «Zeus did not Commit Incest with His Mother. An Interpretation of Column XXVI of the Derveni Papyrus», ZPE 168, p. 27-39.

Casadesús, F., 2008, “El papiro de Derveni”, in A. Bernabé, F. Casadesús (eds.), Orfeo y la tradición órfica. Un reencuentro, Madrid, p. 459-494.

Dorandi, T., 2010, «Diogene Laerzio, Epicuro e gli editori di Epicuro e di Diogene Laerzio», Eikasmos 21, p. 273-301.

Dorandi, T., 2013, "Diogene Laerzio e la storia della filosofia antica. Con qualche considerazione di un editore", in C. Rossitto, A. Coppola, F. Biasutti (eds.), Aristotele e la storia, Padova, p. 185-203.

Frede, M., 2007, «On the Unity and the Aim of the Derveni Text», Rhizai 1, p. 9-33.

JAnko, R., 2001, «The Derveni Papyrus (Diagoras of Melos, Apopyrgizontes logoi?): A New Translation», CP 96, p. 1-32.

Janko, R., 2002, «The Derveni Papyrus: An Interim Text», ZPE 141, p. 1-62.

Jourdan, F., 2003, Le Papyrus de Derveni, Paris.

Kotwick, M.E., 2016, «Notes on PDerveni Cls. XV, XIX, XXII, and XXIV», ZPE 197, p. 1-4.

Kотwicк, M.E., 2017, Der Papyrus von Derveni, eingeleitet, übersetzt und kommentiert, basierend auf einem griechischen Text von R. Janko, Berlin - Boston.

Koтwick, M.E., 2019, "Anoetoi amyetoi: Allegorical Interpretation in the Derveni Papyrus and Plato's Gorgias», CPh 114, p. 173-196.

Kouremenos, T., 2006: cf. KPT 2006.

KPT, 2006, The Derveni Papyrus, edited with introduction and commentary by T. Kouremenos, G. M. Parássoglou, K. Tsantsanoglou, Firenze.

LAKs, A., 2007, Review of KPT 2006, Rhizai 4, p. 153-162.

Laks, A., Most, G. W., 1997, “A Provisional Translation of the Derveni Papyrus”, in IID. (eds.), Studies on the Derveni Papyrus, Oxford, p. 93-128.

Laks, A., Most, G. W., 2016, Early Greek Philosophy, vol. VI: Later Ionians and Athenian Thinkers, part 1, Cambridge (Mass.) - London.

LAPINI, W., 2015, L'Epistola a Erodoto e il bios di Epicuro in Diogene Laerzio. Note testuali, esegetiche e metodologiche, Roma.

LAPINI, W., 2018(a), "Fattori fuorvianti e tutele negli errori ermeneutici e negli errori di copia: repliche moderne di fenomeni antichi”, in G. CRImi, L. MARCozzi (eds.), «Tutto il lume de la spera nostra». Studi per Marco Ariani, Roma, p. 239-253.

LAPINI, W., 2018(b), «Fra protagonismo del demonico e diacosmesi in fieri: in margine a un recente libro sul Papiro di Derveni», Aegyptus 98, p. 37-69.

McKirahan, R. D., 2010², Philosophy Before Socrates. An Introduction with Texts and Commentary, Indianapolis - Cambridge.

Merkelbach, R., 1967, «Der orphische Papyrus von Derveni», ZPE 1, p. 21-32.

OвbInk, D., 1994, «A Quotation of the Derveni Papyrus in Philodemus' On Piety», CE 24, p. 111-135.

Оввіnк, D., 1997, “Cosmology as Initiation vs. the Critique of Orphic Mysteries”, in Laks, Most 1997, p. 39-54.

Oвbink, D., 2003, “Allegory and Exegesis in the Derveni Papyrus: The Origin of Greek Scholarship", in G. R. Boys-Stones (ed.), Metaphor, Allegory and the Classical Tradition, Oxford, p. 177-188.

Obвink, D., 2010, “Early Greek Allegory», in R. Copeland, P. T. Struck (eds.), The Cambridge Companion to Allegory, Cambridge, p. 15-25.

PIAno, V., 2016(a), «P. Derveni III-VI: una riconsiderazione del testo», ZPE 197, p. 5-16. 
Piano, V., 2016(b), Il Papiro di Derveni tra religione e filosofia, Firenze.

Ramelli, I., 2007, Allegoristi dell'età classica. Opere e frammenti, introduzione di R. Radice, Milano.

Rangos, S., 2007, «Latent Meaning and Manifest Content in the Derveni Papyrus», Rhizai 1, p. 35-75.

Rusten, J. S., 1985, «Interim Notes on the Papyrus from Derveni», HSCPh 89, p. 121-140.

Salamone, O., 2016, Le Papyrus de Derveni de la formation du cosmos à la genèse des mots, introduction, édition critique, traduction, notes et étude monographique des fragments du papyrus, Marseille.

Sedley, D., Bastianini, G., 1995, “Commentarium in Platonis Theaetetum”, in CPF, vol. III, Firenze, p. 221-562.

Tortorelli GHidini, M., 2006, Figli della terra e del cielo stellato, Napoli.

Tosi, R., 1988, Studi sulla tradizione indiretta dei classici greci, Bologna.

TsantsanOGLOU, K., 2006: cf. KPT 2006.

Tsantsanoglou, K., Parássoglou, G. M., 2006: cf. KPT 2006.

Wittwer, R., 1999, “Aspasian Lemmatology”, in A. Alberti, R. W. Sharples (eds.), Aspasius: The Earliest Extant Commentary on Aristotle's Ethics, Berlin - New York, p. 51-84. 